\title{
Farmacovigilancia y COVID-19: una radiografía para evitar una necropsia
}

\author{
Pharmacovigilance and COVID-19: an X-ray to avoid necropsy
}

\author{
Ximena Wester-Pérez 1,a, Eduardo Wester-Pérez 1,b
}

\section{Sr. Editor:}

Durante el año 2019, en el mes de diciembre, se identificó en Wuhan, China, un nuevo coronavirus, el cual se propagó muy rápidamente, generando la actual pandemia de SARS-CoV-2, comúnmente conocida como COVID-19 ${ }^{(1)}$, la cual ha impactado con gran repercusión en la población, por su letalidad y su rápida transmisión, y también en la economía mundial.

La acelerada expansión de esta pandemia ocasionó zozobra en la población por todas las consecuencias que acarrea. Este temor, sumado a la ignorancia y ausencia de preparación acerca de cómo afrontar una emergencia sanitaria por parte de la población y del personal de salud, los llevó a tomar medidas drásticas como la automedicación. Esta última es consecuencia directa de la gran difusión de supuestas "medidas curativas" en redes sociales, a las cuales la mayoría de ciudadanos tienen acceso $y$, sin comprobar si son verdaderas o siquiera eficaces, las aplican para cuidar su salud. Estas al ser usadas a largo plazo y sin un estricto control originaron graves daños en el organismo e inclusive llegaron a producir la muerte (2).

En el Perú, debido a la inesperada llegada de la COVID-19 y su rápida propagación, se pudieron observar las deficiencias que existían para hacer frente a un estado de emergencia, generando desconcierto en la población. Además, la inexperiencia en combatirla llevó a experimentar con numerosos fármacos al inicio de la pandemia, algunos de los cuales se materializaron gravemente en la población, ocasionando reacciones desfavorables en el organismo y un fatal desenlace ${ }^{(3)}$.

Según la norma técnica del Ministerio de Salud (MINSA) para pacientes infectados por COVID-19, emitida en abril del año 2020, describe algunos fármacos para el tratamiento de esta infección como lo son hidroxicloroquina, cloroquina, azitromicina, lopinavir/ritonavir (limitado nivel de evidencia), ivermectina (este último aún en estudio), etc. ${ }^{(4)}$. Sin embargo, debido al descaecimiento de la población, la escasez de vigilancia sobre la efectividad de estos medicamentos y, sobre todo, de su rápida propagación en las redes sociales, se optó por la automedicación de dichas drogas, las cuales generaron numerosas repercusiones en el organismo por las múltiples interacciones que presentan.

\footnotetext{
${ }^{1}$ Universidad Católica Santo Toribio de Mogrovejo, Lambayeque-Perú.

${ }^{a}$ Estudiante de Medicina Humana

${ }^{\mathrm{b}}$ Estudiante de Derecho
} 
Es así como durante el presente año se han reportado diversos eventos asociados al uso de estas "medidas", tales como trastornos del ritmo cardíaco manifestados en el electrocardiograma con una prolongación del intervalo QT, asociado al uso de hidroxicloroquina y azitromicina, lo cual eventualmente conducía a la muerte ${ }^{(5)}$. Además, cabe resaltar las repercusiones ligadas al consumo o aplicación de dióxido de cloro, en particular irritación de boca, esófago, pero principalmente trastornos hematológicos, cardiovasculares y renales ${ }^{(6)}$. También hacer notar el uso de ciertos anticoagulantes (warfarina, enoxaparina, etc.) como "profilaxis"(7) para una infección por COVID-19, cuyos principales efectos adversos son hemorragias y trombocitosis ${ }^{(8)}$, y junto a antiparasitarios como la ivermectina, incrementaban e incluso agravaban el riesgo de estas reacciones en la salud de la población ${ }^{(9)}$.

De este modo, se refrenda la trascendencia de la farmacovigilancia en el país, la cual se encarga de detectar, evaluar, comprender y prevenir los efectos adversos de los fármacos o cualquier otro inconveniente que se suscite con estos (10). Esta disciplina, actualmente, representa un reto sobre todo porque debe identificar cuáles son los fármacos que podrían tener algún beneficio o perjuicio en el manejo de la infección por COVID-19, pero fundamentalmente el peligro que estos podrían representar vinculado a su uso desmedido.

Así pues, por todo lo descrito, se pone en evidencia las carencias de nuestro sistema de farmacovigilancia y de salud pública, el cual era crucial frente a esta pandemia, no logrando desarrollarse del modo en que un país con más de 32 millones de habitantes ${ }^{(11)}$ lo exige.

Finalmente, debemos remarcar la ineludible labor que debe desempeñar el Ministerio de Salud, el cual debe trabajar en cooperación con todos los ministerios, pero con énfasis con los ministerios de Transportes y Comunicaciones, de la Mujer y Poblaciones Vulnerables, del Interior y Relaciones Exteriores. Todo esto a fin de que, a través de un cruce de información se logren identificar las zonas donde se ha aumentado la venta y uso de estos fármacos de forma irregular, así también para enfatizar esta vigilancia en zonas vulnerables, donde es más probable la asimetría informativa y otros engaños a la población, sin dejar de resaltar la labor que deben desempeñar la Policía Nacional y Fuerzas Armadas para hacer cumplir las disposiciones del Gobierno Central y de las Gerencias Regionales de Salud que se encuentran descentralizadas en todo el Perú.

Fuentes de financiamiento: Autofinanciado.

Conflictos de interés: Los autores declaran no tener conflictos de interés.

\section{REFERENCIAS BIBLIOGRÁFICAS}

1. Vellas C, Delobel P, De Souto Barreto P, Izopet J. COVID-19, Virology and Geroscience: A Perspective. J Nutr Heal Aging [Internet]. 2020 [cited 2020 Nov 22] ;24(7):685-91. Available from: https://www.ncbi.nlm.nih. gov/pmc/articles/PMC7301052/

2. Alvarez-Risco A, Mejia CR, Delgado-Zegarra J, Del-Aguila-Arcentales $\mathrm{S}$, Arce-Esquivel AA, Valladares-Garrido MJ, et al. The Peru approach against the COVID-19 infodemic: Insights and strategies. Am J Trop Med Hyg [Internet]. 2020[cited 2021 Mar 11] ;103(2):583-6. Available from: https://www.ajtmh.org/view/journals/tpmd/103/2/article-p583.xml

3. MINSA. Automedicación puede agravar salud de pacientes con COVID-19. 2020 Aug 31 [cited 2021 Mar 11];2-3. Available from: https:// www.gob.pe/institucion/minsa/noticias/297083-automedicacion-puede-agravar-salud-de-pacientes-con-covid-19

4. Ministerio de Salud (MINSA). Prevención Diagnóstico y Tratamiento de personas afectadas por COVID-19 en el Perú [Internet]. Lima, Perú; 2020 [cited 2020 Feb 19]. p. 44. Available from: https://cdn.www.gob.pe/ uploads/document/file/582567/Prevención__Diagnóstico_y_Tratamiento_de_personas_afectadas_por_COVID-19_en_el_Perú_.PDF

5. Chorin E, Wadhwani L, Magnani S, Dai M, Shulman E, Nadeau-Routhier $C$, et al. QT interval prolongation and torsade de pointes in patients with COVID-19 treated with hydroxychloroquine/azithromycin. Hear Rhythm [Internet]. 2020 [cited 2020 Nov 22] ;17(9):1425-33. Available from: https://doi.org/10.1016/j.hrthm.2020.05.014

6. Organización Panamericana de la salud. La OPS no recomienda tomar productos que contengan dióxido de cloro, clorito de sodio, hipoclorito de sodio o derivados, 16 de julio del 2020 [Internet]. Perú; 2020 [cited 2020 Nov 22]. p. 6-6. Available from: https://fi-admin.bvsalud.org/document/ view/j9fm9\%0Ahttps://search.bvsalud.org/global-literature-on-novel-coronavirus-2019-ncov/resource/en/covidwho-677289

7. Seguro Social de Salud. Anticoagulación parenteral en el tratamiento de pacientes con COVID-19. [Internet]. Lima, Perú; 2020. Available from: http://docs.bvsalud.org/biblioref/2020/05/1096029/rb-13-aines-editado270320-Im.pdf

8. Instituto de evaluación de tecnologías en salud e investigación. Consideraciones de seguridad en el uso enoxaparina en pacientes con COVID-19 [Internet]. Vol. 191, Thrombosis Research. 2020. p. 145-7. Available from: http://www.essalud.gob.pe/ietsi/pdfs/farmacoytecno/ covid_19/CS_FV10_considerac_seguridad_uso_heparinas_pacientes COVID_19.pdf

9. Instituto de evaluación de tecnologías en salud e investigación. Consideraciones de seguridad en el uso ivermectina en pacientes con COVID-19 [Internet]. Essalud. Lima, Perú; 2020. p. 2. Available from: http://www. essalud.gob.pe/ietsi/pdfs/farmacoytecno/covid_19/CS_FV10_considerac_seguridad_uso_heparinas_pacientes_COVID_19.pdf

10. OMS. Indicadores De Farmacovigilancia: Un manual páctico para la evaluación de los sistemas de farmacovigilancia [Internet]. Perú: Organización mundial de la salud; 2019 [cited 2020 Nov 22]. p. 87. Available from: http://www.digemid.minsa.gob.pe/UpLoad/UpLoaded/PDF/Farmacovigilancia/Indicadores/indicadores_2014.pdf

11. INEI. Población Peruana 2020 [Internet]. Instituto Nacional de Estadística Perú. Perú; 2020 [cited 2020 Nov 22]. p. 1-50. Available from: https:// www.inei.gob.pe/media/MenuRecursivo/publicaciones_digitales/Est/ Lib1743/Libro.pdf 